\title{
Cigarette smoke-induced epithelial expression of WNT-5B: implications for COPD
}

Irene H. Heijink ${ }^{1,2,3}$, Harold G. de Bruin ${ }^{1}$, Robin Dennebos ${ }^{1}$, Marnix R. Jonker ${ }^{1}$, Jacobien A. Noordhoek ${ }^{1}$, Corry-Anke Brandsma ${ }^{1,3}$, Maarten van den Berge ${ }^{2,3}$ and Dirkje S. Postma 2,3

Affiliations: 'University of Groningen, University Medical Center Groningen, Dept of Pathology and Medical Biology, Groningen, The Netherlands. ${ }^{2}$ University of Groningen, University Medical Center Groningen, Dept of Pulmonology, Groningen, The Netherlands. ${ }^{3}$ University of Groningen, University Medical Center Groningen GRIAC Research Institute, Groningen, The Netherlands.

Correspondence: Irene H. Heijink, University Medical Center Groningen, Hanzeplein 1, 9713 GZ, Groningen, The Netherlands. E-mail: h.i.heijink@uumcg.nl

ABSTRACT Wingless/integrase-1 (WNT) signalling is associated with lung inflammation and repair, but its role in chronic obstructive pulmonary disease (COPD) pathogenesis is unclear. We investigated whether cigarette smoke-induced dysregulation of WNT-5B contributes to airway remodelling in COPD.

We analysed WNT-5B protein expression in the lung tissue of COPD patients and (non)smoking controls, and investigated the effects of cigarette smoke exposure on WNT-5B expression in COPD and control-derived primary bronchial epithelial cells (PBECs). Additionally, we studied downstream effects of WNT-5B on remodelling related genes fibronectin, matrix metalloproteinase (MMP)-2, MMP-9 and SnaiI in BEAS-2B and air-liquid interface (ALI)-cultured PBECs.

We observed that airway epithelial WNT-5B expression is significantly higher in lung tissue from COPD patients than controls. Cigarette smoke extract significantly increased mRNA expression of WNT-5B in COPD, but not control-derived PBECs. Exogenously added WNT-5B augmented the expression of remodelling related genes in BEAS-2B cells, which was mediated by transforming growth factor (TGF)- $\beta$ / Smad3 signalling. In addition, WNT-5B upregulated the expression of these genes in ALI-cultured PBECs, particularly PBECs from COPD patients.

Together, our results provide evidence that exaggerated WNT-5B expression upon cigarette smoke exposure in the bronchial epithelium of COPD patients leads to TGF- $\beta /$ Smad3-dependent expression of genes related to airway remodelling.

@ERSpublications

Cigarette smoke enhances WNT-5B expression in COPD airway epithelium, causing expression of airway remodelling genes http://ow.ly/ZxiAy

This article has supplementary material available from erj.ersjournals.com

Received: Sept 152015 | Accepted after revision: March 102016 | First published online: April 282016

Support statement: This study was supported by the Dutch Royal Academy of Sciences (KNAW). Funding information for this article has been deposited with FundRef.

Conflict of interest: Disclosures can be found alongside the online version of this article at erj.ersjournals.com

Copyright OERS 2016 


\section{Introduction}

Chronic obstructive pulmonary disease (COPD) is a life-threatening disease with a worldwide increase in morbidity and mortality, it is characterised by chronic airway inflammation, irreversible airflow limitation and accelerated lung function decline [1]. Abnormal tissue repair upon cigarette smoking is an important pathophysiological feature of the disease, leading to increased extracellular matrix (ECM) deposition and small airway wall thickening. These structural changes cannot be cured and there is an urgent need for a better understanding of the underlying mechanisms.

The airway epithelium forms the first barrier to inhaled cigarette smoke, separating the underlying mesenchyme from the environment. Cigarette smoke causes epithelial damage, which is thought to play a pivotal role in airway remodelling. Especially when the epithelial layer is damaged, epithelial cells produce growth factors (e.g. transforming growth factor (TGF)- $\beta$ ) that act on underlying tissue to regulate repair and remodelling processes [2]. TGF- $\beta$ is a key mediator of airway remodelling in COPD [2] and a well-known inducer of epithelial-to-mesenchymal transition (EMT), a process involved in tissue repair. During EMT, epithelial markers like E-cadherin are lost and mesenchymal markers, e.g. fibronectin, matrix metalloproteinases (MMPs) and Snail, are gained. Cigarette smoke has been shown to induce EMT in airway epithelium in a TGF- $\beta$-dependent manner [3].

Wingless/integrase-1 (WNT) signalling [4], a pathway critically involved in the regulation of lung development, is re-activated upon tissue damage and inflammation in the lungs [5]. The autocrine and paracrine effects of WNT ligands are mediated by frizzled receptors (FZD), leading to transcription of ECM proteins, MMPs and other remodelling genes [6]. Canonical WNT signalling results in $\beta$-catenin-mediated transcription, an important aspect of EMT. In addition, WNT ligands can induce gene transcription through noncanonical pathways, including $\mathrm{p} 38, \mathrm{Ca}^{2+} /$ nuclear factor of activated $\mathrm{T}$-cell (NFAT) and RhoA-dependent signalling [7, 8]. Of interest, WNT signalling has been implicated in lung epithelial injury and repair processes $[5,9,10]$ and dysregulated expression of WNT genes has been observed in COPD lung tissue and airway epithelium [11-13].

The role of WNT signalling in the development and progression of airway remodelling in COPD is still largely unknown. In the current study, we hypothesised that aberrant epithelial expression of WNT ligands upon cigarette smoke exposure contributes to airway remodelling in COPD. We show that WNT-5B expression is increased upon cigarette smoke exposure in bronchial epithelial cells, particularly in cells from COPD patients, and that WNT-5B induces TGF- $\beta /$ Smad3-dependent transcription of EMT/remodelling genes.

\section{Methods}

\section{Subjects}

Lung tissue was derived from lungs of seven smoking COPD patients and control lungs of seven smokers with normal lung function and eight nonsmokers (table 1). The tissue was derived from tumour resection surgery left-over lung tissue, taken far distant from the tumour and checked for abnormalities by experienced pathologists. Primary bronchial epithelial cells (PBECs) were obtained by protease digestion, as described previously [14], from tracheobronchial tissue of nine ex-smokers with severe COPD undergoing lung transplantation (table 1), and six non-COPD lung donors about whom no further information was available. The study protocol was consistent with the Research Code of the University Medical Center Groningen (www.umcg.nl/SiteCollectionDocuments/English/Researchcode/UMCG-Researchcode,\%20basic \%20principles\%202013.pdf) and national ethical and professional guidelines ("Code of conduct; Dutch federation of biomedical scientific societies"; www.federa.org). In addition, PBECs were obtained by fibre-optic bronchial brushing according to standard guidelines in five smokers with COPD and six

TABLE 1 Characteristics of the subjects from whom lung tissue was obtained

\begin{tabular}{lcccc} 
& Control nonsmoker & Control smoker & COPD stage II $^{\#}$ & COPD stage IV \# $^{\#}$ \\
\hline Subjects $\mathbf{n}$ & 8 & 7 & 7 & 9 \\
Female \% & 63 & 43 & 29 & 80 \\
Age years & $67(46-81)$ & $56(47-61)$ & $56(47-70)$ & $57(44-61)$ \\
Smoking status & Nonsmoker & Current & Current & Ex-smoker \\
FEV $\%$ predicted & $103(85-114)$ & $94(91-104)$ & $67(56-80)$ & $20(16-60)$ \\
\hline
\end{tabular}

Data are presented as median (range), unless otherwise stated. COPD: chronic obstructive pulmonary disease; FEV1: forced expiratory volume in 1s. \#: COPD patients were included based on the Global Initiative for Chronic Obstructive Lung Disease standard [1]; $\eta_{:} \geqslant 10$ pack-years. 
TABLE 2 Characteristics of the subjects undergoing bronchial brushings

\begin{tabular}{lcc} 
& Control nonsmoker & COPD stage II $^{\#}$ \\
\hline Subjects $\mathbf{n}$ & 6 & 5 \\
Female \% & 17 & 0 \\
Age years & $61(49-73)$ & $66(64-69)$ \\
Smoking status & Nonsmoker & Current $^{\text {" }}$ \\
FEV $\%$ predicted & $113(89-129)$ & $65(49-79)$ \\
\hline
\end{tabular}

Data are presented as median (range), unless otherwise stated. COPD: chronic obstructive pulmonary disease; FEV1: forced expiratory volume in $1 \mathrm{s.}{ }^{\#}$ : COPD patients were included based on the Global Initiative for Chronic Obstructive Lung Disease standard [1]; ๆ: $\geqslant 10$ pack-years.

nonsmoking controls in a study approved by the Medical Ethics Committee of our centre (table 2). All participants gave their written informed consent.

\section{Immunohistochemistry}

Lung tissue from seven smokers with COPD, seven control smokers and eight nonsmokers was embedded in paraffin, used for immunohistochemistry and quantified by automated computer-assisted image analysis as described in the online supplementary material.

\section{Cell culture}

BEAS-2B cells were cultured on collagen-coated flasks in RPMI medium (Biowhittaker, Verviers, Belgium)/ $10 \%$ fetal calf serum. PBECs were obtained as described earlier and cultured in hormonally supplemented bronchial epithelium growth medium (BEGM; Lonza, Walkersville, MD, USA) in collagen/fibronectin-coated flasks. PBECs were stored in liquid nitrogen before use in passage three. For experimentation, cells were grown in duplicates in 24-well plates until $80-90 \%$ confluence and serum/hormonally deprived overnight. For mucociliary-differentiated epithelium, PBECs were grown at the air-liquid interface (ALI) for 2 weeks, as described previously [15].

\section{Cell stimulation}

Prior to stimulation, cells were transfected with small interfering (si)RNA or reporter constructs or pretreated with/without specific inhibitors as described in the online supplementary material. Subsequently, cells were exposed to 5-10\% cigarette smoke extract (CSE), which does not affect cell viability [16], $5 \mathrm{ng} \cdot \mathrm{mL}^{-1} \mathrm{TGF}-\beta 1$ and/or recombinant human WNT-5B (5-500 ng.mL ${ }^{-1}, \mathrm{R} \& \mathrm{D}$ systems, Minneapolis, MN, USA) for 6 or $24 \mathrm{~h}$. Cell-free supernatants were collected and cells were harvested in TRIreagent (MRC Inc, Cincinatti, OH, USA) for RNA isolation or in Laemmli buffer for cell lysate preparation. See the online supplementary material for methods on cell transfection, concentration of inhibitors, CSE preparation, RNA isolation, cDNA synthesis, real-time PCR, western blotting, MMP-2 measurement and dual luciferase assay.

\section{Statistical analysis}

Data were analysed using the nonparametric rank-sum Mann-Whitney U-test between subject groups, nonparametric Wilcoxon-signed rank test for paired observations within subject groups, and repeated measurements ANOVA with Bonferroni's multiple comparison test or t-test for analysis of paired observations within the cell line, based on the assumption of a normal distribution.

\section{Results}

Increased WNT-5B protein expression in lung tissue upon smoke exposure in vivo We previously reported that human bronchial epithelial cells express substantial levels of WNT-4, WNT-5A, WNT-5B and WNT-7B mRNA, and that cigarette smoke exposure specifically upregulates WNT-5B mRNA expression in vitro [11]. To study whether cigarette smoking increases airway epithelial WNT-5B expression in vivo, we compared the protein expression of WNT-5B in lung tissue from nonsmokers to control smokers and smokers with COPD using immunohistochemistry. Importantly, WNT-5B was strongly expressed in lung tissue from smokers with COPD, with significantly higher airway epithelial expression than nonsmokers as well as control smokers, without a significant difference between the latter two groups (figure 1). This suggests that higher WNT-5B expression is not smoking related but disease related, or that airway epithelium from COPD patients is especially susceptible to cigarette smoke-induced upregulation of WNT-5B.

\section{Cigarette smoke upregulates WNT-5B expression in airway epithelium}

To investigate this further, we studied the effect of cigarette smoke exposure on WNT-5B expression in primary airway epithelial cells from COPD patients and nonsmoking controls in vitro. We previously 

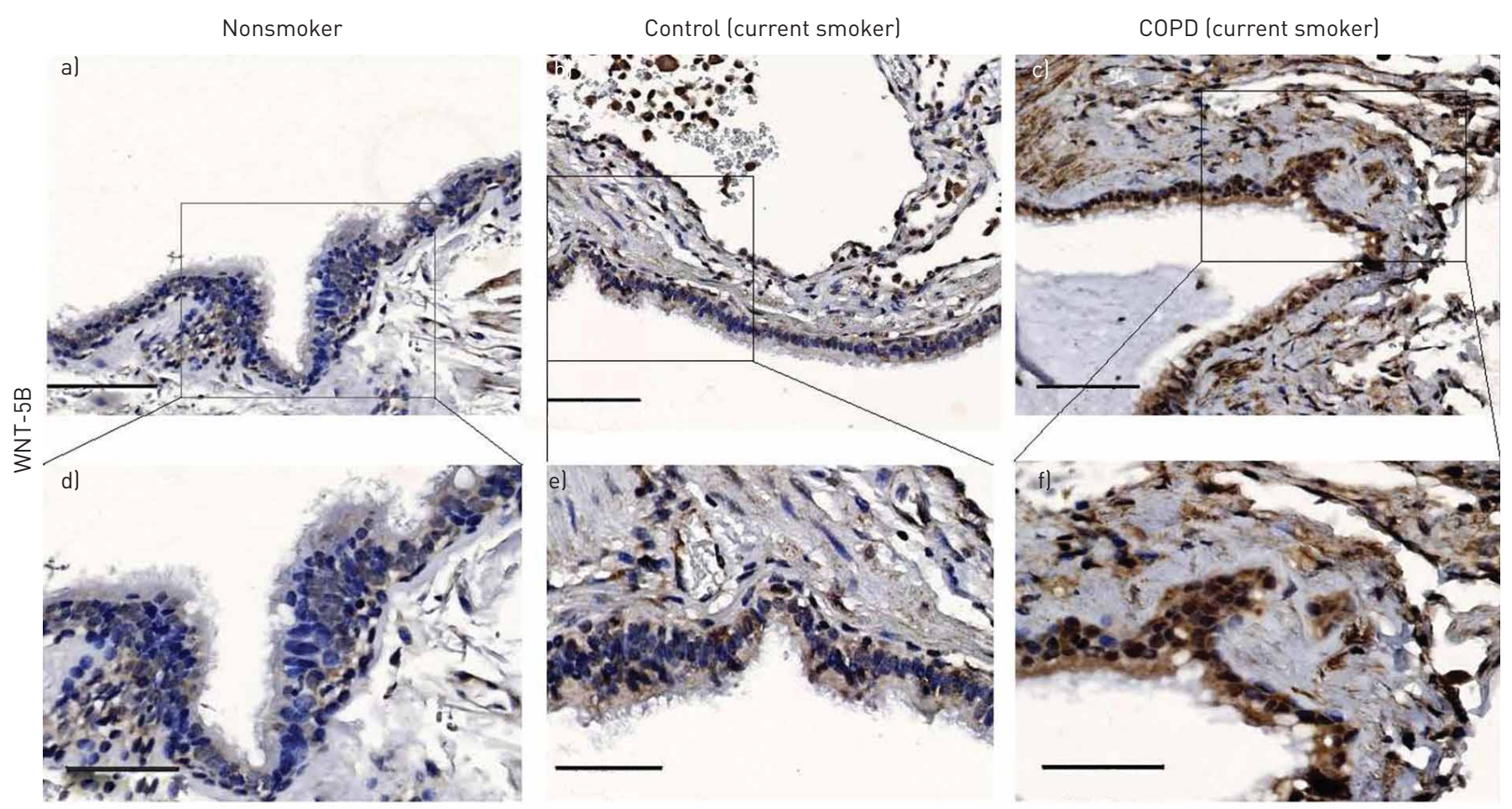

\section{COPD}
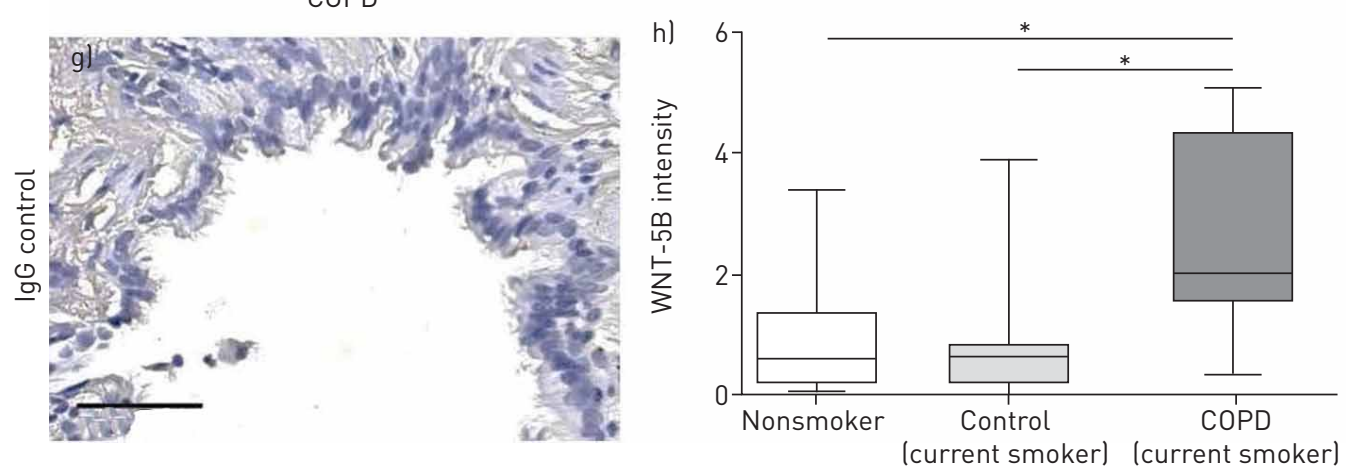

FIGURE 1 Increased WNT-5B protein expression in lung tissue upon smoke exposure in vivo. Lung tissue was obtained from a, d) eight non-smokers, b, el seven control smokers and c, f) seven smoking chronic obstructive pulmonary disease (COPD) patients in Global Initiative for Chronic Obstructive Lung Disease stage II (table 1). Immunohistochemistry was performed for WNT-5B (a-f) or lgG control antibody (g) and h) staining intensity in the bronchial epithelial layer was quantified using computer-assisted imaging. $a-c)$ Scale bars=80 $\mu \mathrm{m}$; $d-g$ ) $s c a l e$ bars $=50 \mu m$. *: $p<0.05$ between the indicated values tested using the Mann-Whitney U-test.

reported that PBECs from COPD patients express higher mRNA levels of WNT-4 compared with nonsmokers and control smokers at baseline, without differential expression of WNT-5B [11]. Again, we did not observe significant differences in baseline expression of WNT-5B mRNA in COPD- and control-derived PBECs (figure 2a). 6-h exposure to 5\% CSE significantly enhanced WNT-5B expression in PBECs from five current smokers and three ex-smokers with COPD, whereas no significant CSE-induced increase was present in control-derived PBECs (figure 2a). At the protein level, we studied the effect of both $5 \%$ and $10 \%$ CSE. Although we did not observe significant differences, the lower concentration of CSE had a maximal effect in COPD-derived cells, while in control-derived cells the maximal effect was observed at the higher concentration (figure $2 \mathrm{~b}$ ) Together, these data suggest that COPD epithelium is more prone to upregulate WNT-5B expression upon cigarette smoke exposure. In contrast, 5\% CSE induced an equally strong increase in expression of the redox-sensitive gene haem oxygenase in PBECs from COPD patients and controls (figure S1).

Because of the limited number of COPD-derived PBECs, we used a human bronchial cell line to further investigate the role of WNT-5B in airway epithelial remodelling mechanistically. We used BEAS-2B, since this cell line is susceptible to undergo CSE-induced EMT [17]. Here, 10\% CSE significantly increased 

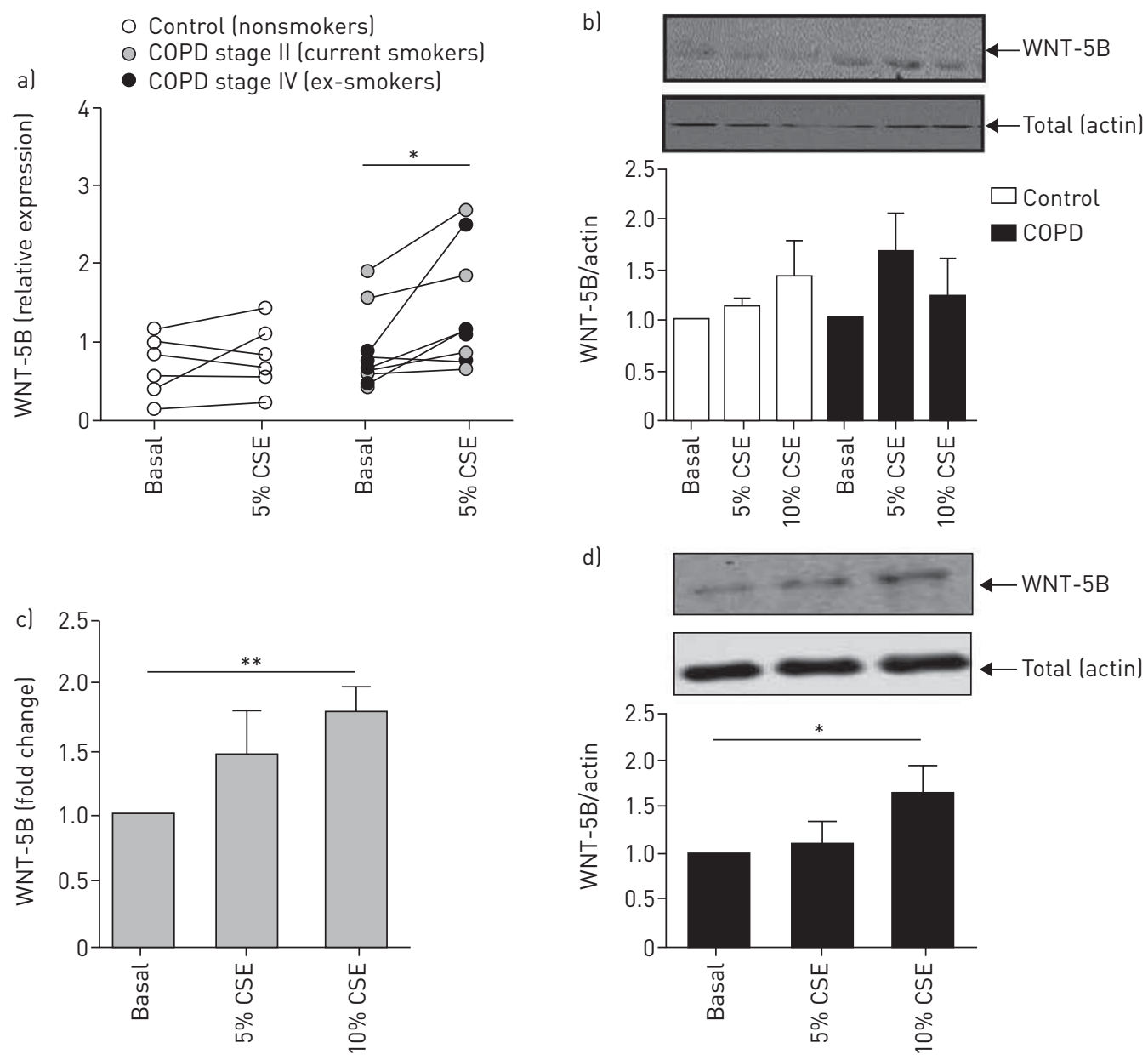

FIGURE 2 Exposure to cigarette smoke extract (CSE) upregulates WNT-5B expression in airway epithelium. a) Primary bronchial epithelial cells (PBECs) from six nonsmoking controls (table 2), five current smoking COPD patients with Global Initiative for Chronic Obstructive Lung Disease (GOLD) stage II (table 2) and three ex-smoking COPD patients with GOLD stage IV (table 1) were exposed with/without CSE for $6 \mathrm{~h}$. WNT-5B mRNA expression was related to the expression of the house keeping genes $\beta$-2-microglobulin ( $\beta 2 \mathrm{~m})$ and peptidylprolyl isomerase A (PPIA). mRNA levels are expressed as fold change compared with the unstimulated control value $\left(2^{-\Delta \Delta C t}\right)$. Medians are indicated. b) PBECs from six non-COPD controls and four ex-smoking COPD patients with stage GOLD stage IV (table 1) were incubated with or without $5 \%$ and $10 \%$ CSE for $24 \mathrm{~h}$. Total cell lysates were prepared and WNT-5B was detected by Western blotting. Actin was used as the loading control. Densitometry was performed and levels were related to actin levels. A representative blot and the mean \pm SEM WNT-5B/actin ratios are depicted. C) BEAS-2B cells were incubated with or without $5 \%$ and $10 \%$ CSE for $6 \mathrm{~h}$, RNA was isolated and cDNA synthesised. Expression of WNT-5B was detected by quantitative PCR and related to the expression of the house keeping genes $\beta 2 \mathrm{~m}$ and PPIA. mRNA levels are expressed as mean \pm SEM fold change compared with the unstimulated control value $\left(2^{-\Delta \Delta C t}, n=4-7\right)$. d) BEAS-2B cells were incubated with or without $5 \%$ and $10 \%$ CSE for $24 \mathrm{~h}$. Total cell lysates were prepared and WNT-5B was detected by Western blotting. Actin was used as the loading control. Densitometry was performed and levels were related to actin levels. A representative blot and the mean \pm SEM WNT-5B/actin ratios are depicted $(n=5) .{ }^{*}: p<0.05$; and ${ }^{* *}: p<0.01$, as tested by the non-parametric Wilcoxon-signed rank test for the primary cells and the t-test for paired observations in BEAS-2B cells.

WNT-5B mRNA expression, whereas 5\% CSE did not (figure 2c). The upregulatory effect was confirmed at the protein level (figure $2 \mathrm{~d}$ ).

\section{WNT-5B regulates the expression of remodelling markers in BEAS-2B}

Next, we assessed the effects of exogenously added WNT-5B on markers of cigarette smoke-induced airway remodelling in COPD, i.e. fibronectin, MMP-2, MMP-9 and E-cadherin [18-21]. 6-h exposure to $500 \mathrm{ng} \cdot \mathrm{mL}^{-1}$, but not 5 or $50 \mathrm{ng} \cdot \mathrm{mL}^{-1}$, WNT-5B significantly increased fibronectin mRNA expression in BEAS-2B cells (figure 3a). WNT-5B (500 ng. $\mathrm{mL}^{-1}$ ) also significantly increased the expression of MMP-2, MMP-9 and the E-cadherin repressor Snail, and reduced E-cadherin mRNA (figure 3b). These effects were confirmed at the protein level for fibronectin and E-cadherin (figure 3c). Furthermore, WNT-5B significantly increased the release of MMP-2 protein (figure 3d), while levels of MMP-9 were below the 

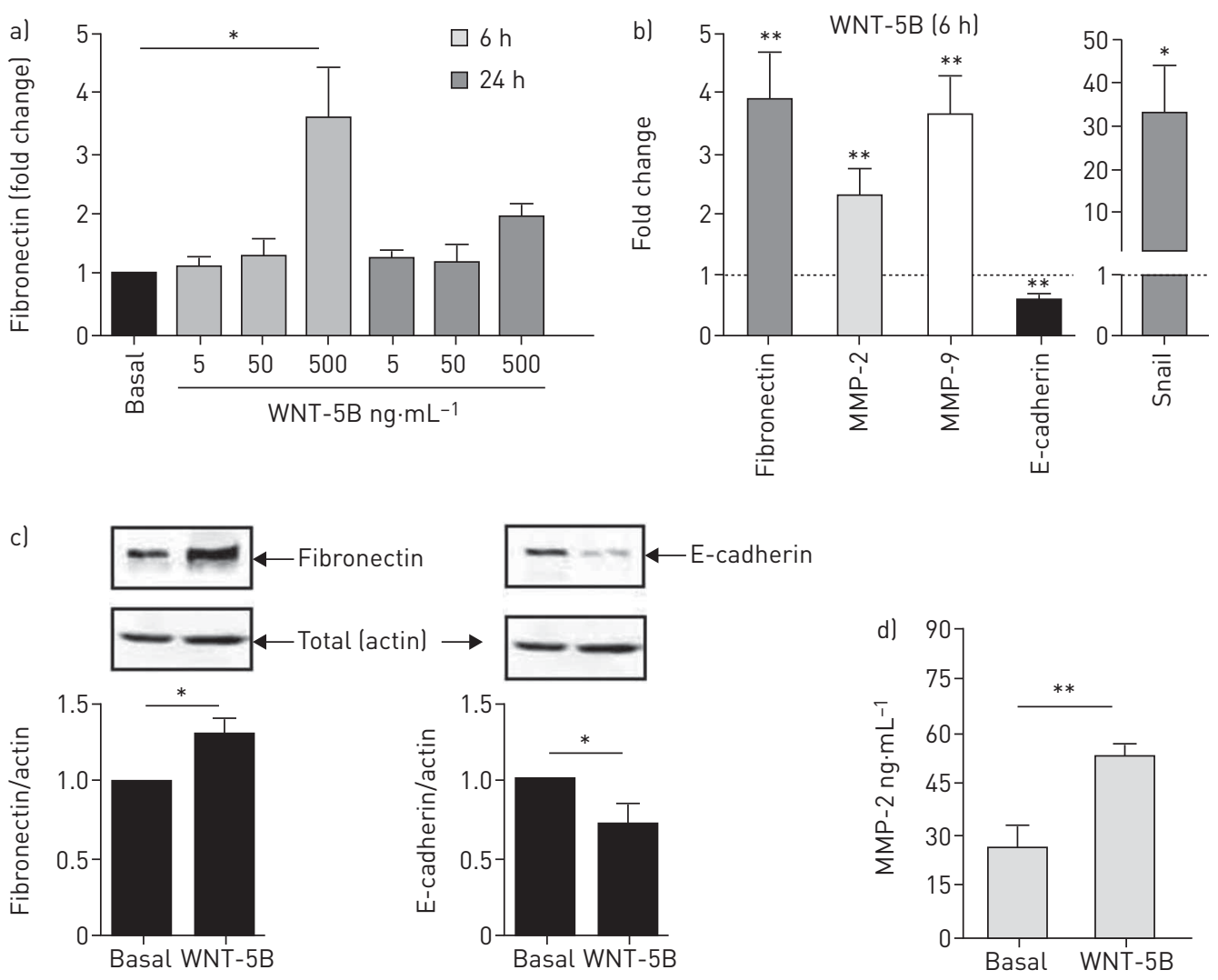

FIGURE 3 WNT-5B is upregulated by transforming growth factor (TGF)- $\beta$ and induces the expression of remodelling genes in BEAS-2B cells. BEAS-2B cells were incubated with or without WNT-5B $\left(500 \mathrm{ng} \cdot \mathrm{mL}^{-1}\right.$, unless otherwise indicated). a) After 6 and $24 \mathrm{~h}$, fibronectin mRNA expression was related to the expression of the house keeping genes $\beta$-2-microglobulin $(\beta 2 \mathrm{~m})$ and peptidylprolyl isomerase A (PPIA). mRNA levels are expressed as mean \pm SEM fold change compared with the unstimulated control value $\left(2^{-\Delta \Delta C t}, n=4-7\right)$. b) After $6 \mathrm{~h}, \mathrm{mRNA}$ expression of fibronectin, matrix metalloproteinase (MMP)-2, MMP-9, E-cadherin and Snail expression was related to the expression of the house keeping genes $\beta 2 m$ and PPIA. mRNA levels are expressed as mean \pm SEM fold change compared with the unstimulated control value $\left(2^{-\Delta \Delta C t}, n=9-14\right)$. c) Total cell lysates were prepared after $24 \mathrm{~h}$ and fibronectin and E-cadherin were detected by Western blotting. Actin was used as the loading control. Densitometry was performed and levels were related to actin levels. A representative blot and the mean \pm SEM ratios are depicted $(n=4)$. d) MMP-2 levels were detected in cell-free supernatants after $24 \mathrm{~h}$ of treatment using Luminex. Absolute levels (mean \pm SEM) are shown $(n=6) .{ }^{*}: p<0.05$; and ${ }^{* *}: p<0.01$, between the indicated values or compared with the unstimulated control tested using the t-test for paired observations ( panels a, $c$ and d) or repeated measures ANOVA ( panel b).

detection limit of the assay. Part of the secreted MMP-2 was active, as indicated by the reduction in MMP-2 levels upon extraction of active MMPs with tumour necrosis factor protease inhibitor 2 (TAPI-2)-labelled beads (figure S2).

\section{WNT-5B induces activation of Smad3 and p38 signalling in BEAS-2B}

To elucidate the signalling pathways involved in these effects of WNT-5B, we used reporter constructs containing TCF/LEF-1 or NFAT and AP- 1 binding sites for canonical WNT/ $\beta$-catenin and non-canonical WNT signalling, respectively, in BEAS-2B cells. We also included a Smad-binding element (SBE) reporter, given the implications of TGF- $\beta$, a downstream molecule Smad3, for EMT [3, 22]. WNT-5B significantly increased SBE activity, while it had no effect on $\beta$-catenin (figure $4 \mathrm{a}, \mathrm{b}$ ), NFAT and AP-1 activation (figure S3). To assess whether Smad was indeed activated by WNT-5B, we studied Smad3 phosphorylation and observed a significant increase upon WNT-5B exposure (figure 4c, d). In addition, WNT-5B significantly increased phosphorylation of the non-canonical signalling molecule p38.

WNT-5B-induced expression of remodelling-related genes depends on TGF- $\beta / S$ mad3 signalling in BEAS-2B

We next examined whether p38 and Smad3 mediate the effect of WNT-5B on gene transcription using selective inhibitors. The Smad3 inhibitor SIS3 significantly reduced the effect of WNT-5B on fibronectin 

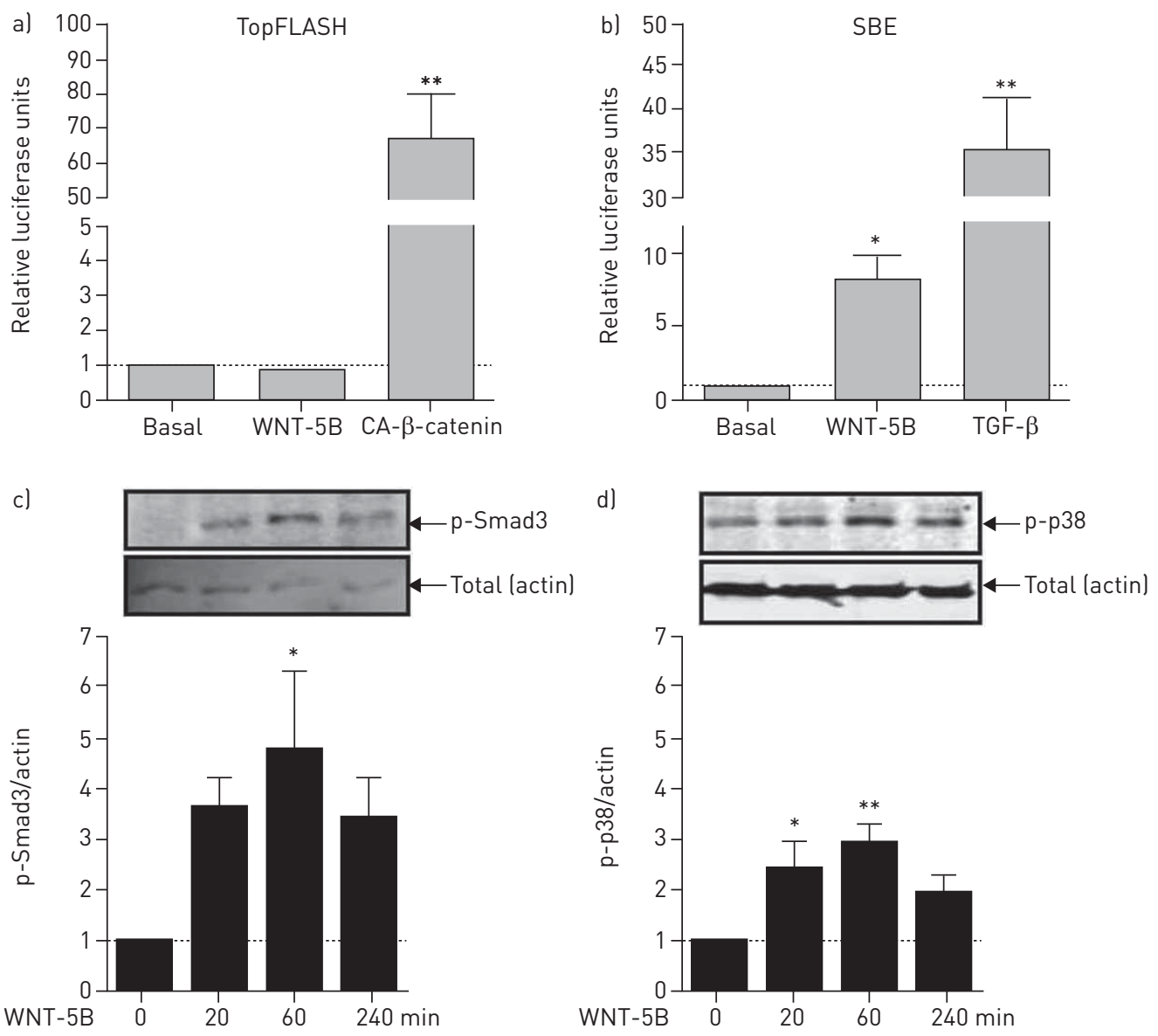

FIGURE 4 WNT-5B does not induce activity of the $\beta$-catenin-driven reporter TopFLASH, but activates a Smad binding element (SBE) driven reporter and increases phospho-Smad3 (p-Smad3) and phospho-p38 (p-p38) levels in BEAS-2B cells. a, b) Cells were grown overnight and transfected with a) the TopFLASH reporter (200 ng) with/ without $200 \mathrm{ng}$ constitutively active $\beta$-catenin (CA- $\beta$-catenin) construct (positive control for $\beta$-catenin signalling) or b) with the SBE reporter (200 ng) and the thymidine kinase-driven Renilla luciferase vector pRL-TK (20 ng) as the internal control, and stimulated with/without $500 \mathrm{ng} \cdot \mathrm{mL}^{-1}$ WNT-5B or $5 \mathrm{ng} \cdot \mathrm{mL}^{-1}$ transforming growth factor (TGF)- $\beta$ (positive control for Smad signalling) for $24 \mathrm{~h}$. Luciferase activity was determined, values were normalised (reporter/Renilla ratio) and expressed as means \pm SEM $(n=5)$. c, d) Serum-deprived cells were incubated with $500 \mathrm{ng} \cdot \mathrm{mL}^{-1}$ WNT-5B for 0-240 min. Total cell lysates were prepared and c) p-Smad3 and d) p-p38 were detected by Western blotting. Actin was used as the loading control. Densitometry was performed and levels were related to actin levels. Representative blots and the mean \pm SEM ratios are depicted $(n=4) .{ }^{*}: p<0.05$; and **: $p<0.01$ between the indicted value and the unstimulated control tested using t-tests for paired observations (panels $a$ and $b$ ) or repeated measures ANOVA (panels $\mathrm{c}$ and $\mathrm{d}$ ).

mRNA expression, and a similar effect was observed for the p38 mitogen-activated protein kinase (MAPK) inhibitor SB203580, albeit to a smaller extent (figure 5a). Smad3 and p38 MAPK are both downstream targets of TGF- $\beta$ signalling and are activated upon binding of TGF- $\beta$ to its receptor (T $\beta$ R)II/activin receptor-like kinase (Alk)5 [23]. To assess involvement of TGF- $\beta$ /T $\beta$ RII signalling in the transcriptional effects of WNT-5B, we used the TßRII/Alk5 inhibitor SB431542, and this completely abrogated the effect of WNT-5B on fibronectin mRNA expression (figure $5 \mathrm{~b}$ ). Given the relatively short time that this took to occur, we anticipate that the effects of WNT-5B are exerted by activation of the TGF- $\beta$ receptor and downstream signalling rather than by increasing de novo synthesis of TGF- $\beta$, although WNT-5B significantly increased TGF- $\beta$ mRNA expression (figure S4). TGF- $\beta$ is synthesised and secreted as a latent complex, in which it is incorporated into the ECM. Upon tissue damage, TGF- $\beta$ can be converted from the latent into the active form to bind its receptor by several mechanisms, including changes in the ECM and proteolytic activation by plasmin and MMP-2/9 [24]. Of interest, WNT-5B-induced fibronectin mRNA expression was almost completely abrogated by the use of the broad-spectrum MMP inhibitor TAPI-2 (figure 5c). Thus, WNT-5B may induce TGF- $\beta$ activation and downstream signalling by increasing active MMP levels. In further support of a role for TGF- $\beta$ signalling in the effects of WNT-5B, the upregulatory effect of WNT-5B on fibronectin was blocked by addition of a neutralising TGF- $\beta$ antibody (figure 5b). Furthermore, exogenously added TGF- $\beta 1$ mimicked the effects of WNT-5B, with a 

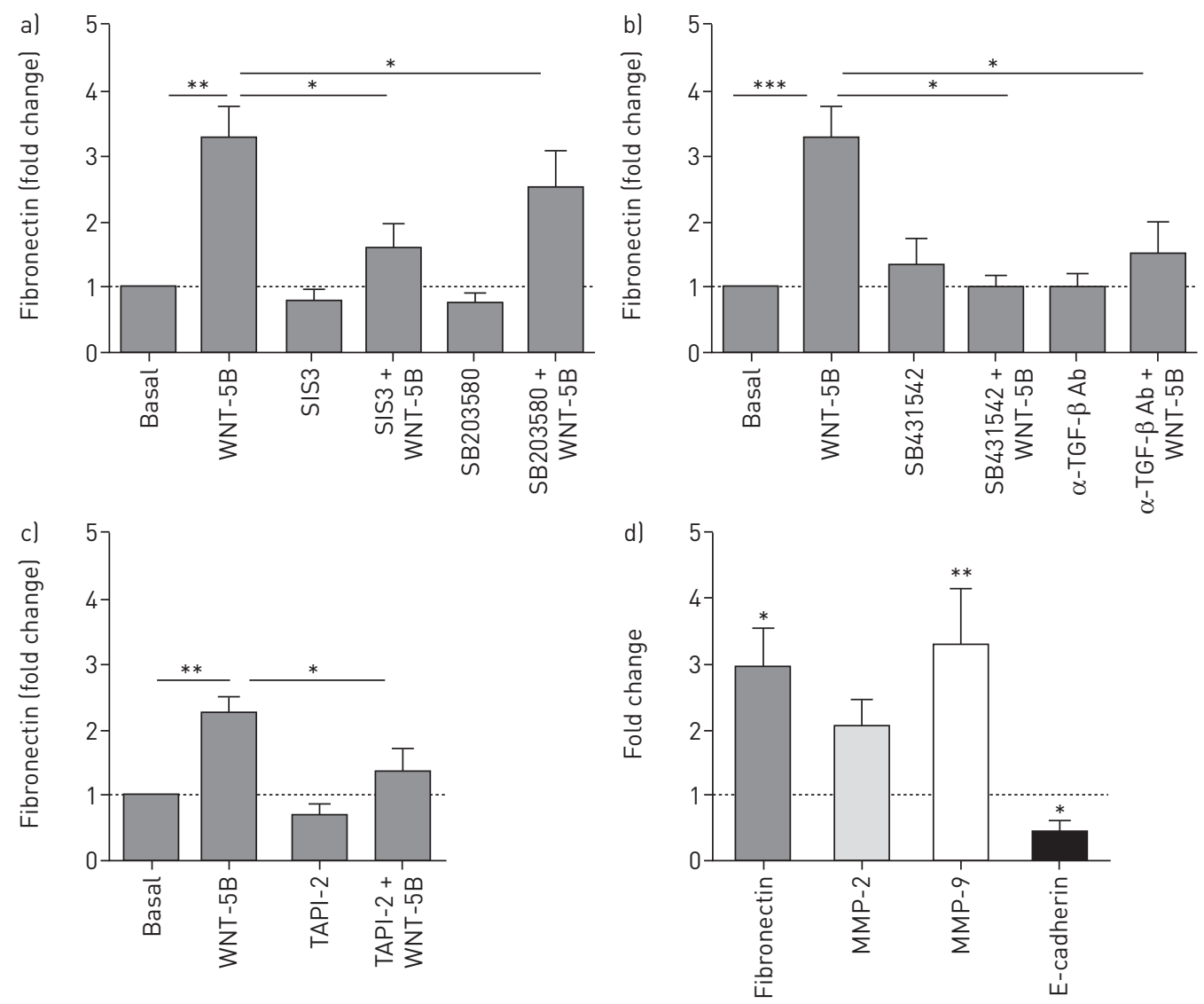

FIGURE 5 The effect of WNT-5B on remodelling genes is mediated by transforming growth factor (TGF)- $\beta / \mathrm{Smad} 3$ signalling in BEAS-2B cells. a) Cells ( $n=7-9$ ) were pre-treated with $3 \mu \mathrm{M}$ SIS3 or $1 \mu \mathrm{M}$ SB203580 for 30 min and subsequently exposed to $500 \mathrm{ng} \cdot \mathrm{mL}^{-1}$ WNT-5B for $6 \mathrm{~h}$. b) Cells $(\mathrm{n}=6-7)$ were pre-treated with $20 \mu \mathrm{M}$ SB431542 or $5 \mu \mathrm{g} \cdot \mathrm{mL}^{-1}$ neutralising TGF- $\beta$ antibody ( $\alpha-$ TGF- $\beta$ Ab) for $60 \mathrm{~min}$ and subsequently exposed to $500 \mathrm{ng} \cdot \mathrm{mL}^{-1}$ WNT-5B for $6 \mathrm{~h}$. Fibronectin mRNA expression was related to the expression of the house keeping genes $\beta$-2-microglobulin $(\beta 2 \mathrm{~m})$ and peptidylprolyl isomerase A (PPIA). c) Cells $(\mathrm{n}=5)$ were pretreated with $20 \mu \mathrm{M}$ TAPI-2 for 30 min and subsequently exposed to $500 \mathrm{ng} \cdot \mathrm{mL}^{-1}$ WNT-5B for $6 \mathrm{~h}$. Fibronectin mRNA expression was related to the expression of the house keeping genes $\beta 2 \mathrm{~m}$ and PPIA. d) Cells ( $\mathrm{n}=9$ ) were incubated with/without $5 \mathrm{ng} \cdot \mathrm{mL}^{-1}$ TGF- $\beta$ for $6 \mathrm{~h}$; fibronectin, matrix metalloproteinase (MMP)-2, MMP-9 and E-cadherin mRNA expression was related to the expression of the house keeping genes $\beta 2 \mathrm{~m}$ and PPIA. mRNA levels are expressed as the mean \pm SEM fold change compared with the unstimulated control value $\left(2^{-\Delta \Delta C t}\right){ }^{*}: p<0.05 ;{ }^{* *}: p<0.01$; and ${ }^{* * *}: p<0.001$ between the indicated values as tested by repeated measures ANOVA (panels $a-c$ ) or t-test for paired observations (panel d).

downregulatory effect on E-cadherin mRNA expression and an upregulatory effect on fibronectin, MMP-2 and MMP-9 mRNA expression (figure 5d).

\section{WNT-5B effects in PBECs from controls and COPD patients}

To enhance the translational relevance of our findings in BEAS-2B cells, we assessed whether WNT-5B induces similar effects in PBECs. WNT-5B strongly and significantly increased the expression of fibronectin, MMP-2 and MMP-9 mRNA in PBECs derived from three severe COPD patients and three non-COPD controls (figure 6a), with equal effects of WNT-5B in cells from COPD patients and controls. Next, we used ALI-differentiated PBECs from six COPD patients and six non-COPD controls to more closely reflect the situation in vivo [25]. Baseline levels of WNT-5B (figure S5), fibronectin, MMP-2, MMP-9 and SnaiI (not shown) did not significantly differ in ALI-cultured PBECs from controls and COPD patients. When WNT-5B was added to the apical side to reflect stimulation in an autocrine fashion, a significant increase in MMP-2 mRNA expression was observed in both groups (figure 6c), similar to the effects observed in submerged cultured PBECs. Furthermore, WNT-5B did not significantly upregulate fibronectin (figure 6b), MMP-9 (figure 6d) and SnaiI (figure 6e) expression in PBECs from controls, whereas it significantly increased fibronectin and Snail expression in PBECs from COPD patients, with a trend in the similar direction for MMP-9.

Together, our results suggest that WNT-5B may contribute to airway remodelling in COPD patients by promoting TGF- $\beta$ signalling in a positive feedback loop. 

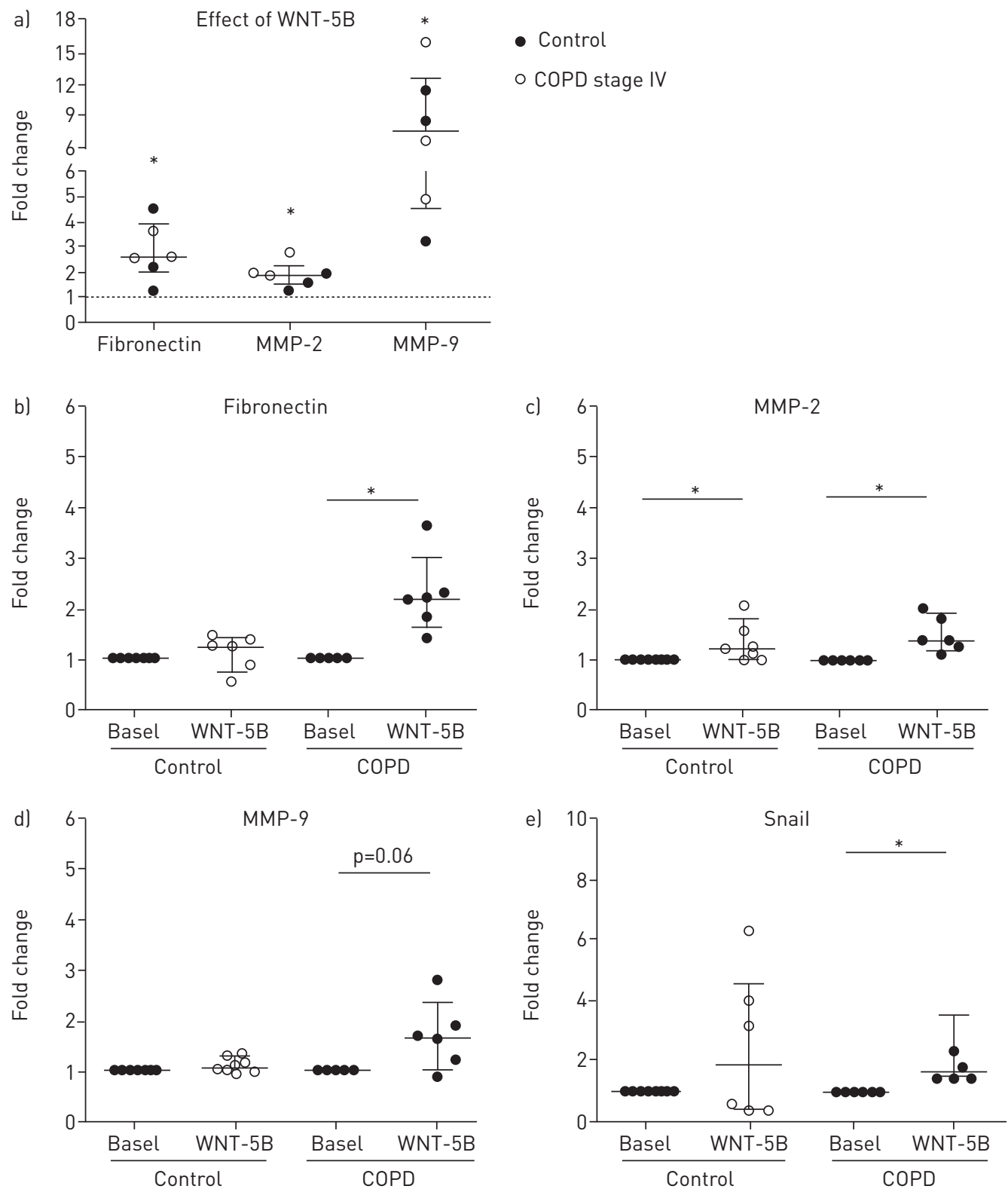

FIGURE 6 Effects of WNT-5B on primary bronchial epithelial cells (PBECs) from chronic obstructive pulmonary disease (COPD) patients and controls. a) PBECs were obtained from tracheobronchial tissue of three non-COPD controls and three ex-smoking COPD patients with Global Initiative for Chronic Obstructive Lung Disease (GOLD) stage IV disease (table 1). Cells were exposed to $500 \mathrm{ng} \cdot \mathrm{mL}^{-1}$ WNT-5B for $6 \mathrm{~h}$. Fibronectin, matrix metalloproteinase (MMP)-2 and MMP-9 mRNA expression was related to the expression of the house keeping genes $\beta$-2-microglobulin $(\beta 2 \mathrm{~m})$ and peptidylprolyl isomerase $A$ (PPIA). b-e) PBECs were derived from tracheobronchial tissue of six non-COPD controls and six ex-smoking COPD patients with GOLD stage IV disease (table 1). Cells were grown at the air-liquid interface (ALI) for 2 weeks, washed and apically exposed to 500 $\mathrm{ng} \cdot \mathrm{mL}^{-1}$ WNT-5B in BEBM/DMEM or BEBM/DMEM alone for $6 \mathrm{~h}$. Fibronectin, MMP-2, MMP-9 and Snail mRNA expression was related to the expression of the house keeping genes $\beta 2 \mathrm{~m}$ and PPIA, and mRNA levels are expressed as fold change compared with the unstimulated control value $\left(2^{-\Delta \Lambda c^{t}}\right)$. Medians and interquartile ranges are indicated. *: p<0.05 between the indicated values, as tested using the Mann-Whitney U-test for analysis between groups and the non-parametric Wilcoxon-signed rank test for analysis within groups.

\section{Discussion}

Our data in human lung tissue and epithelial cells indicate an important role for WNT-5B in cigarette smoke-induced airway epithelial remodelling in COPD. Airway epithelial expression of the WNT-5B protein was significantly higher in lung tissue from smokers with COPD than in tissue from control smokers and nonsmokers, without a significant difference between the latter two. Accordingly, cigarette smoke exposure increased WNT-5B expression in bronchial epithelial cells, particularly in COPD-derived epithelium. This 
confirms our hypothesis that cigarette smoking leads to aberrant WNT-5B expression in COPD airways. In line with our hypothesis, WNT-5B upregulated epithelial expression of fibronectin, MMP-2, MMP-9 and Snail, potentially contributing to airway remodelling in COPD. Finally, our data show for the first time that these downstream effects of WNT-5B are mediated by TGF- $\beta /$ Smad3-dependent signalling.

We provide evidence that airway epithelium from COPD patients is more prone to upregulate WNT-5B expression in response to cigarette smoking, as supported by the in vitro observation that CSE only induces a significant increase in WNT-5B expression in COPD-derived but not control-derived bronchial epithelial cells. In line with these findings, our group previously demonstrated that fibroblasts from COPD patients are more prone to upregulate WNT-5B expression upon TGF- $\beta$ stimulation than fibroblasts from controls matched for smoking history [26]. This may contribute to the susceptibility to develop structural changes in the airways, as we have shown that WNT-5B acts in a positive feedback loop to facilitate TGF- $\beta$ signalling, promoting the expression of the EMT markers fibronectin, MMP-2, MMP-9 and SnaiI. Recently, a role for EMT in COPD airway remodelling and peribronchial fibrosis has been proposed [4]. Milara et al. [3] reported loss of epithelial characteristics, e.g. E-cadherin expression, in cultured small airway epithelial cells from COPD patients [3, 22]. SoHAL et al. [27] observed increased expression of various EMT markers, including MMP-9, in large airway epithelium of COPD patients. YANG et al. [28] reported that expression of SnaiI exon variant c.353T $>$ C, which attenuates its ability to promote EMT, is associated with a decreased risk to develop COPD. Furthermore, higher fibronectin expression, which was particularly found in the small airways of COPD patients compared with nonsmoking and smoking controls, was reported to associate with lower lung function [28]. Similarly, higher lung expression of MMP-2 [20] and higher serum and sputum levels of MMP-9 associate with lower lung function in COPD [21]. Thus, we propose that WNT-5B may be involved in remodelling of the airway epithelium and contribute to the pathophysiology of COPD. Therefore, it will be of importance to study the mechanism of CSE-induced WNT-5B expression in future studies.

Our data show that WNT-5B expression promotes TGF- $\beta / S m a d 3$ signalling in bronchial epithelial cells, a pathway known to induce the expression of mesenchymal genes during EMT, including fibronectin [18, 19]. In line with our findings, both WNT-3 and WNT-5B have recently been described as critical factors secreted from TGF- $\beta$-induced mesenchymal cancer cells, inducing an invasive epithelial phenotype along with the induction of EMT [23]. Our data indicate a role for non-canonical WNT signalling in the effects of WNT-5B in airway epithelium, as WNT-5B activated Smad3 and p38 instead of $\beta$-catenin. Accordingly, the induction of WNT-5B expression was shown to be accompanied by p38 and Smad2/3 signalling and the expression of EMT markers in a different epithelial cell type, tubular epithelium of the fibrotic kidney [29]. Of note, both Smad3 and p38 are downstream molecules of TGF- $\beta$ receptor activation [30] and our data indicate that the effects of WNT-5B are mediated by TGF- $\beta$ receptor downstream signalling.

We used relatively high concentrations of WNT-5B. Of note, equally high concentrations were previously used to induce differentiation of human embryonic stem cells [23, 31], and we anticipate that recombinant WNT-5B may lack the specific post-translational modifications required to optimally activate its receptor(s). The short time frame in which WNT-5B induced TGF- $\beta$ downstream signalling suggests that WNT-5B increases the bioavailability of TGF- $\beta$ for its receptor, although the exact mechanism requires further investigation. In line with a role for proteolytic activation of latent TGF- $\beta$, e.g. by MMP-2 [32], we observed that WNT-5B increases levels of (partly active) MMP-2 in human bronchial epithelium. In future studies, it will be of interest to assess if and how WNT-5B increases MMP activity, e.g. by $\mathrm{Ca}^{2+}$-dependent activation of proteases [25], leading to the observed activation of TGF- $\beta / S m a d 3$ signalling in bronchial epithelial cells.

Interestingly, specific single-nucleotide polymorphisms in SMAD3 were reported to associate with COPD in a Chinese population $[33,34]$. Smad3 null mice were resistant to TGF- $\beta$ and bleomycin-induced fibrosis, indicating that Smad3 signalling may have detrimental effects in fibrotic disease [35]. By contrast, Smad3 null mice developed spontaneous age-related airspace enlargement, consistent with emphysema [36]. Similar to TGF- $\beta /$ Smad3 signalling, we speculate that WNT signalling may have a dual role in COPD. We and others have shown increased WNT-4 expression in bronchial epithelial cells [36] and bronchial biopsies [11] of COPD patients compared with controls. In an elastase-induced emphysema mouse model, however, reduced expression of WNT (target) genes, e.g. WNT-10B, WNT-2, FZD1 and axin1/2, was observed [37]. Here, WNT/ $\beta$-catenin activation attenuated airspace enlargement [12], pointing towards a protective role of the WNT pathway in peripheral lung tissue destruction, while our data suggest that WNT-5B signalling may exert detrimental effects in the airways of COPD patients, promoting airway remodelling.

Together, our data show that cigarette smoke increases WNT-5B expression in airway epithelial cells, especially those from COPD individuals, leading to increased TGF- $\beta /$ smad 3 signalling with a downstream increase in remodelling markers. Thus, targeting WNT-5B may constitute a novel therapeutic approach in the treatment of airway remodelling in COPD patients. 


\section{References}

1 Pauwels RA, Buist AS, Calverley PM, et al. Global strategy for the diagnosis, management, and prevention of chronic obstructive pulmonary disease. NHLBI/WHO Global Initiative for Chronic Obstructive Lung Disease (GOLD) Workshop summary. Am J Respir Crit Care Med 2001; 163: 1256-1276.

2 Takizawa $\mathrm{H}$, Tanaka M, Takami K, et al. Increased expression of transforming growth factor-betal in small airway epithelium from tobacco smokers and patients with chronic obstructive pulmonary disease (COPD). Am J Respir Crit Care Med 2001; 163: 1476-1483.

3 Milara J, Peiró T, Serrano A, et al. Epithelial to mesenchymal transition is increased in patients with COPD and induced by cigarette smoke. Thorax 2013; 68: 410-420.

4 Baarsma HA, Spanjer AI, Haitsma G, et al. Activation of WNT/ $\beta$-catenin signaling in pulmonary fibroblasts by TGF- $\beta 1$ is increased in chronic obstructive pulmonary disease. PLoS One 2011; 6: e25450.

5 Königshoff M, Eickelberg O. WNT signaling in lung disease: a failure or a regeneration signal? Am J Respir Cell Mol Biol 2010; 42: 21-31.

6 Clevers H. Wnt/beta-catenin signaling in development and disease. Cell 2006; 127: 469-480.

7 Arnsdorf EJ, Tummala P, Jacobs CR. Non-canonical Wnt signaling and N-cadherin related beta-catenin signaling play a role in mechanically induced osteogenic cell fate. PLoS One 2009; 4: e5388.

8 Ma L, Wang HY. Mitogen-activated protein kinase p38 regulates the Wnt/cyclic GMP/Ca ${ }^{2+}$ non-canonical pathway. J Biol Chem 2007; 282: 28980-28990.

9 Crosby LM, Waters CM. Epithelial repair mechanisms in the lung. Am J Physiol Lung Cell Mol Physiol 2010; 298 : L715-L731.

10 Königshoff M, Kramer M, Balsara N, et al. WNT1-inducible signaling protein-1 mediates pulmonary fibrosis in mice and is upregulated in humans with idiopathic pulmonary fibrosis. J Clin Invest 2009; 119: 772-787.

11 Heijink IH, de Bruin HG, van den Berge M, et al. Role of aberrant WNT signalling in the airway epithelial response to cigarette smoke in chronic obstructive pulmonary disease. Thorax 2013; 68: 709-716.

12 Kneidinger N, Yildirim AO, Callegari J, et al. Activation of the WNT/beta-catenin pathway attenuates experimental emphysema. Am J Respir Crit Care Med 2011; 183: 723-733.

13 Wang R, Ahmed J, Wang G, et al. Down-regulation of the canonical Wnt $\beta$-catenin pathway in the airway epithelium of healthy smokers and smokers with COPD. PLoS One 2011; 6: e14793.

14 Hackett TL, Shaheen F, Johnson A, et al. Characterization of side population cells from human airway epithelium. Stem Cells 2008; 26: 2576-2585.

15 Heijink IH, Postma DS, Noordhoek JA, et al. House dust mite-promoted epithelial-to-mesenchymal transition in human bronchial epithelium. Am J Respir Cell Mol Biol 2010; 42: 69-79.

16 Heijink IH, Brandenburg SM, Postma DS, et al. Cigarette smoke impairs airway epithelial barrier function and cell-cell contact recovery. Eur Respir J 2012; 39: 419-428.

17 Wang Q, Wang $\mathrm{H}$, Zhang $\mathrm{Y}$, et al. Activation of uPAR is required for cigarette smoke extract-induced epithelial-mesenchymal transition in lung epithelial cells. Oncol Res 2013; 21: 295-305.

18 Brajer B, Batura-Gabryel H, Nowicka A, et al. Concentration of matrix metalloproteinase-9 in serum of patients with chronic obstructive pulmonary disease and a degree of airway obstruction and disease progression. J Physiol Pharmacol 2008; 59: Suppl. 6, 145-152.

19 Vernooy JH, Lindeman JH, Jacobs JA, et al. Increased activity of matrix metalloproteinase-8 and matrix metalloproteinase-9 in induced sputum from patients with COPD. Chest 2004; 126: 1802-1810.

20 Annoni R, Lanças T, Yukimatsu Tanigawa R, et al. Extracellular matrix composition in COPD. Eur Respir J 2012; 40: $1362-1373$.

21 Baraldo S, Bazzan E, Zanin ME, et al. Matrix metalloproteinase-2 protein in lung periphery is related to COPD progression. Chest 2007; 132: 1733-1740.

22 Gohy ST, Hupin C, Fregimilicka C, et al. Imprinting of the COPD airway epithelium for dedifferentiation and mesenchymal transition. Eur Respir J 2015; 45: 1258-1272.

23 Hackett TL, Warner SM, Stefanowicz D, et al. Induction of epithelial-mesenchymal transition in primary airway epithelial cells from patients with asthma by transforming growth factor-betal. Am J Respir Crit Care Med 2009; 180: 122-133.

24 Bakin AV, Rinehart C, Tomlinson AK, et al. p38 mitogen-activated protein kinase is required for TGFbeta-mediated fibroblastic transdifferentiation and cell migration. J Cell Sci 2002; 115: 3193-3206.

25 Hayashi H, Sakai T. Biological significance of local TGF- $\beta$ activation in liver diseases. Front Physiol 2012; 3: 12.

26 Heijink IH, Noordhoek JA, Timens W, et al. Abnormalities in airway epithelial junction formation in chronic obstructive pulmonary disease. Am J Respir Crit Care Med 2014; 189: 1439-1442.

27 Sohal SS, Reid D, Soltani A, et al. Evaluation of epithelial mesenchymal transition in patients with chronic obstructive pulmonary disease. Respir Res 2011; 12: 130.

28 Yang L, Yang X, Ji W, et al. Effects of a functional variant c.353T $>\mathrm{C}$ in snail on risk of two contextual diseases. Chronic obstructive pulmonary disease and lung cancer. Am J Respir Crit Care Med 2014; 189: 139-148.

29 Kato S, Hayakawa Y, Sakurai H, et al. Mesenchymal-transitioned cancer cells instigate the invasion of epithelial cancer cells through secretion of WNT3 and WNT5B. Cancer Sci 2014; 105: 281-289.

30 Kim MK, Maeng YI, Sung WJ, et al. The differential expression of TGF- $\beta 1$, ILK and wnt signaling inducing epithelial to mesenchymal transition in human renal fibrogenesis: an immunohistochemical study. Int J Clin Exp Pathol 2013; 6: 1747-1758.

31 Bhowmick NA, Zent R, Ghiassi M, et al. Integrin beta 1 signaling is necessary for transforming growth factor-beta activation of p38MAPK and epithelial plasticity. J Biol Chem 2001; 276: 46707-46713.

32 Nicenboim J, Malkinson G, Lupo T, et al. Lymphatic vessels arise from specialized angioblasts within a venous niche. Nature 2015; 522: 56-61.

33 O'Connell MP, Marchbank K, Webster MR, et al. Hypoxia induces phenotypic plasticity and therapy resistance in melanoma via the tyrosine kinase receptors ROR1 and ROR2. Cancer Discov 2013; 3: 1378-1393.

34 Jiang L, Zhang J, Monticone RE, et al. Calpain-1 regulation of matrix metalloproteinase 2 activity in vascular smooth muscle cells facilitates age-associated aortic wall calcification and fibrosis. Hypertension 2012; 60: $1192-1199$. 
35 Yang IA, Relan V, Wright CM, et al. Common pathogenic mechanisms and pathways in the development of COPD and lung cancer. Expert Opin Ther Targets 2011; 15: 439-456.

36 Gauldie J, Kolb M, Ask K, et al. Smad3 signaling involved in pulmonary fibrosis and emphysema. Proc Am Thorac Soc 2006; 3: 696-702.

37 Durham AL, McLaren A, Hayes BP, et al. Regulation of Wnt4 in chronic obstructive pulmonary disease. FASEB J 2013; 27: 2367-2381. 